\title{
Image quality assessment of pediatric chest and abdomen CT by deep learning reconstruction
}

\author{
Haesung Yoon \\ Severance Hospital \\ Jisoo Kim \\ Severance Hospital \\ Hyun Ji Lim \\ Severance Hospital \\ Mi-Jung Lee ( $\nabla$ mjl1213@yuhs.ac) \\ Severance Hospital
}

\section{Research Article}

Keywords: pediatric, CT, image quality, deep learning, iterative reconstruction

Posted Date: April 20th, 2021

DOI: https://doi.org/10.21203/rs.3.rs-424298/v1

License: (9) This work is licensed under a Creative Commons Attribution 4.0 International License. Read Full License 


\section{Abstract}

Background Efforts to reduce the radiation dose have continued steadily, with new reconstruction techniques. Recently, image denoising algorithms using artificial neural networks, termed deep learning reconstruction (DLR), have been applied to CT image reconstruction to overcome the drawbacks of iterative reconstruction (IR). The purpose of our study was to compare objective and subjective image quality of DLR and IR on pediatric abdomen and chest CT images.

Methods This retrospective study included pediatric body CT images from February 2020 to October 2020, performed on 51 patients (34 boys and 17 girls; age $1-18$ years). Non-contrast chest CT $(n=16)$, contrast-enhanced chest CT $(n=12)$, and contrast-enhanced abdomen CT $(n=23)$ images were included. Standard $50 \%$ adaptive statistical iterative reconstruction V (ASIR-V) images were compared to images with $100 \%$ ASIR-V and DLR at medium and high strengths. Attenuation, noise, contrast to noise ratio (CNR), and signal to noise (SNR) measurements were performed. Overall image quality, artifacts, and noise were subjectively assessed by two radiologists using a four-point scale (superior, average, suboptimal, and unacceptable). Quantitative and qualitative parameters were compared using repeated measures analysis of variance (ANOVA) with Bonferroni correction and Wilcoxon signed-rank tests.

Results DLR had better CNR and SNR than $50 \%$ ASIR-V in both pediatric chest and abdomen CT images. When compared with 50\% ASIR-V, high strength DLR was associated with noise reduction in non-contrast chest CT (33.0\%), contrast-enhanced chest CT (39.6\%), and contrast-enhanced abdomen CT (38.7\%) with increases in CNR at $149.1 \%, 105.8 \%$ and $53.1 \%$ respectively. The subjective assessment of overall image quality and noise was also better on DLR images $(p<0.001)$. However, there was no significant difference in artifacts between reconstruction methods.

Conclusion Compared with $50 \%$ ASIR-V, DLR improved pediatric body CT images with significant noise reduction. However, artifacts were not improved by DLR, regardless of strength.

\section{Background}

The need for pediatric computed tomography (CT) examinations is constantly increasing despite the "as low as reasonably achievable" principle and concerns of radiation hazards for children. Pediatric body CT, including in emergency rooms and in tumor patients, is an important imaging test in children. With the development of technology, efforts to reduce the radiation dose have continued steadily, with the development and use of iterative reconstruction (IR) as a typical example.

Over the past decade, the IR algorithm has been used to produce high resolution images by decreasing image noise through the use of computational processing, resulting in better image quality with lower radiation dose compared with single reconstructed filtered back projection (FBP) in adults $[1,2]$ and children [3-6]. The recently developed adaptive statistical iterative reconstruction-V (ASIR-V) technique provides a short reconstruction time with better image quality and lower radiation dose than other IR algorithms [7, 8]. However, ASIR-V still does not overcome excessive image smoothing and unnatural 
image appearance. Hybrid IR images that blend IR with FBP can be used to decrease this texture problem, although a trade-off between image noise and image texture occurs [9].

Recently, image denoising algorithms using artificial neural networks, termed deep learning reconstruction (DLR), have been applied to CT image reconstruction to overcome the drawbacks of IR while achieving good image quality [10-15]. However, there have been a limited number of studies evaluating this technique in a small number of children and the technique was only evaluated in abdomen CT images [16-18]. The purpose of our study was to compare objective and subjective image quality of DLR and IR on pediatric abdomen and chest CT images.

\section{Methods}

This was a retrospective study approved by the institutional review board at our institution, and the need for informed consent was waived.

\section{Study population}

We included all consecutive pediatric patients who underwent chest or abdomen CT at our institution between February 2020 and October 2020 with the same CT system (Revolution CT; GE Healthcare), which has a routine protocol including DLR. We retrospectively reviewed 51 patients. There were 34 boys and 17 girls with a mean age of $11.5 \pm 4.6$ years (range: $1-18$ years). Non-enhanced chest CT $(n=16)$, contrast-enhanced chest CT $(n=12)$, and contrast-enhanced abdomen CT $(n=23)$ images were included. Height and weight was recorded at the time of CT examination and BMI was calculated. Body weight group was divided as $<20 \mathrm{~kg}, 20-60 \mathrm{~kg}$, and $>60 \mathrm{~kg}$.

\section{Scanning technique and radiation dose measurements}

All patients were examined using a 256-slice CT (Revolution CT; GE Healthcare). Peak kilovoltage (kVp) was divided in to three groups by weight: $100 \mathrm{kVp}$ for $>40 \mathrm{~kg}, 80 \mathrm{kVp}$ for $15-40 \mathrm{~kg}$, and $70 \mathrm{kVp}$ for $<15$ kg. An automatic dose modulation technique (Smart mA; GE Healthcare) was used with a range of 50200 mAs. The noise index was 33 for abdomen CT and 22 for chest CT. Other parameters used to generate images were as follows: gantry rotation time, $0.35 \mathrm{~s}$; coverage speed, $226.79 \mathrm{~mm} / \mathrm{s}$; pitch, 0.992:1; and slice thickness, $2.5 \mathrm{~mm}$.

Weight-based IV contrast injection was used with settings of 1.5-2.0 ml/kg with a maximum of $100 \mathrm{ml}$, using $300 \mathrm{mg}$ iodine/ml concentration intravenous contrast iobitridol (Xenetix; Laboratoires Guerbet). The contrast was injected through an upper extremity peripheral intravenous line, followed by a saline chaser of $0.5 \mathrm{ml} / \mathrm{kg}$. Injection speed was adjusted for a total injection time of $15 \mathrm{sec}$ or less. For contrastenhanced abdomen CT, a fixed time interval of $60 \mathrm{sec}$ after contrast injection for portal phase without bolus tracking was used. For contrast-enhanced chest CT, a circular region of interest (ROI) was placed at the main pulmonary artery and the CT scan began $4 \mathrm{sec}$ after the threshold attenuation of 100 Hounsfield units $(\mathrm{HU})$ was reached. 
Four axial reconstructions were generated for each patient with a $2.5 \mathrm{~mm}$ slice thickness and $2.5 \mathrm{~mm}$ slice interval according to the standard algorithm: 50\% ASIR-V, 100\% ASIR-V, medium- and high-strength DLR (TrueFidelity; GE Healthcare). We set the blending factors to $50 \%$ and $100 \%$ according to previous experience $[3,4]$. DLR provides three selectable reconstruction strength levels (low, medium, and high) to control the amount of noise reduction with a standard reconstruction kernel. We chose medium and high based on our preliminary experience.

The CT dose index volume (CTDIvol, $\mathrm{mGy}$ ) and dose-length product (DLP, $\mathrm{mGy} \times \mathrm{cm}$ ) of all patients were recorded in both CT examinations. CTDIvol was converted to size-specific dose estimates (SSDE) on the basis of the American Association of Physicists in Medicine Report 204 [19]. Patient-specific dimensions were obtained from axial CT images at the carina on chest CT and at the main portal vein on abdomen $\mathrm{CT}$. We used the sum of anteroposterior and lateral dimensions to determine patient effective diameter and conversion factors. The following equation was used to calculate the effective dose (ED, mSv): $E D=$ $\mathrm{DLP} \times \mathrm{W}_{\mathrm{T}}$ (tissue-weighting factor; variable according to $\mathrm{kVp}$, organ, and age [20]). Tissue-weighting factors of less than $80 \mathrm{kVp}$ are unknown, so a tissue-weighting factor of $80 \mathrm{kVp}$ was adopted for $70 \mathrm{kVp}$ studies.

\section{Quantitative image analysis}

Quantitative analysis of axial images was performed by a board-certified radiologist with 9 years of experience. The mean attenuation (HU) and standard deviation (SD) were measured by manually placing the round $\mathrm{ROI}$ (8-10 $\mathrm{mm}$ in diameter) using a picture archiving and communication system (PACS) workstation (Centricity Radiology RA1000; GE Healthcare) in the mediastinal/soft tissue window setting (window level, $50 \mathrm{HU}$; window width, $350 \mathrm{HU}$ ). On chest CT images, ROls were placed in lung and paraspinal muscles at the level of the carina. On abdomen CT images, ROls were placed in liver, aorta, and paraspinal muscles at the level of the main portal vein on axial images. To obtain reliable measurements for the areas, each ROI was positioned to encompass the homogeneous portion and did not include surrounding structures or vessels. Image noise was defined as the SD of the pixel values obtained from paraspinal muscle. Both contrast- and signal-to-noise ratios (CNR and SNR) were defined as $C N R=\left|H U_{\text {object }}-H U_{\text {muscle }}\right| / S_{\text {noise }}$ and $S N R=H U_{\text {object }} / S_{\text {noise }}[21]$.

\section{Qualitative image analysis}

CT images were independently reviewed by two board-certified pediatric radiologists with 17 and 9 years of experience who were blinded to the clinical findings and the CT reconstruction methods. Images were displayed on the PACS in random order and two radiologists independently recorded their opinions on overall image quality, noise, and motion or beam hardening artifacts. A four-point scale was used: 4 was superior, 3 was average, 2 was suboptimal, and 1 was unacceptable.

\section{Statistical analysis}


All statistical analyses were performed using MedCalc software (version 12.1.0; MedCalc Software). Patient demographic characteristics and dose descriptors (CTDI ${ }_{\mathrm{vo}}$, DLP, SSDE, and ED) are summarized and presented as the mean and SD. Repeated measures ANOVA with pairwise comparisons and Bonferroni correction were performed to compare the reconstructions with respect to attenuation, noise, CNR, and SNR. Wilcoxon signed rank and Cohen kappa tests were performed to compare qualitative evaluation and to assess interobserver agreement. Agreement between reviewers is expressed as $\mathrm{K}$ values: $\mathrm{K}$ values of $0-0.20,0.21-0.40,0.41-0.60,0.61-0.80$, and greater than 0.81 indicated poor, fair, moderate, good, and excellent agreements, respectively. A $p$-value of less than 0.05 was considered statistically significant.

\section{Results}

The mean weight and BMl of the patients were $44.3 \pm 18.9 \mathrm{~kg}$ and $20.3 \pm 5.1 \mathrm{~kg} / \mathrm{m}^{2}$, respectively. Two patients had metallic hardware within the scanned field of view of the CT images. CTDI ${ }_{\text {vol }}$, DLP, SSDE, and ED of chest CT images were 1.3 $\pm 0.5 \mathrm{mGy}$ (range, 0.6-2.5 mGy), $49.0 \pm 26.3 \mathrm{mGy} \times \mathrm{cm}$ (range, 16.5$112.9 \mathrm{mGy} \times \mathrm{cm}$ ), $2.0 \pm 0.6 \mathrm{mGy}$ (range,1.2-3.1 mGy), and 2.2 $\pm 3.2 \mathrm{mSv}$ (range, 0.7-16.1 mSv), respectively. $\mathrm{CTDI}_{\mathrm{vol}}, \mathrm{DLP}, \mathrm{SSDE}$, and ED of abdomen CT images were $1.5 \pm 0.6 \mathrm{mGy}$ (range, 0.4-3.2 $\mathrm{mGy}$ ), $77.9 \pm 35.0 \mathrm{mGy} \times \mathrm{cm}$ (range, 12.6-147.7 mGy×cm), $2.5 \pm 0.9 \mathrm{mGy}$ (range, 0.8-4.7 mGy), and $2.0 \pm$ $0.7 \mathrm{mSv}$ (range, $0.7-3.7 \mathrm{mSv}$ ), respectively.

\section{Quantitative image assessment}

The results of the quantitative image assessment are summarized in Table 1 and Fig. 1. The mean attenuation values between reconstructions were equivalent. 
Table 1

Quantitative image analysis of pediatric CT with different reconstruction techniques in comparison with $50 \%$ ASIR-V.

\begin{tabular}{|c|c|c|c|c|c|c|c|c|}
\hline \multicolumn{2}{|l|}{ Parameters } & \multirow{2}{*}{$\begin{array}{l}\text { ASIR-V } \\
50 \\
\text { hancemen }\end{array}$} & \multirow[t]{2}{*}{ ASIR-V 100} & \multicolumn{2}{|c|}{ DLR-M } & \multicolumn{2}{|c|}{ DLR-H } & \\
\hline \multicolumn{7}{|c|}{ Chest CT without contrast enhancement $(n=16)$} & & \\
\hline \multirow[t]{2}{*}{$\begin{array}{l}\text { Attenuation } \\
(\mathrm{HU})\end{array}$} & lung & $\begin{array}{l}-795.9 \pm \\
91.1\end{array}$ & $\begin{array}{l}-796.2 \pm \\
94.2\end{array}$ & 0.872 & $\begin{array}{l}-797.6 \pm \\
94.3\end{array}$ & 0.872 & $\begin{array}{l}-796.7 \\
\pm 93.9\end{array}$ & 0.872 \\
\hline & $\begin{array}{l}\text { paraspinal } \\
\text { muscle }\end{array}$ & $\begin{array}{l}56.1 \pm \\
11.1\end{array}$ & $\begin{array}{l}54.6 \pm \\
12.1\end{array}$ & 0.552 & $\begin{array}{l}56.3 \pm \\
10.7\end{array}$ & 0.552 & $\begin{array}{l}54.9 \pm \\
9.8\end{array}$ & 0.552 \\
\hline Noise & & $\begin{array}{l}21.8 \pm \\
3.7\end{array}$ & $\begin{array}{l}15.6 \pm \\
10.9\end{array}$ & 0.159 & $\begin{array}{l}20.2 \pm \\
3.8\end{array}$ & 0.455 & $\begin{array}{l}14.6 \pm \\
2.5\end{array}$ & $\begin{array}{l}<.001 \\
0.00\end{array}$ \\
\hline CNR & lung & $\begin{array}{l}11.4 \pm \\
3.7\end{array}$ & $\begin{array}{l}22.9 \pm \\
6.9\end{array}$ & $<.001$ & $\begin{array}{l}25.8 \pm \\
13.3\end{array}$ & $\begin{array}{l}< \\
0.001\end{array}$ & $\begin{array}{l}28.4 \pm \\
11.4\end{array}$ & $\begin{array}{l}<.001 \\
0.00\end{array}$ \\
\hline SNR & lung & $\begin{array}{l}10.7 \pm \\
3.5\end{array}$ & $\begin{array}{l}21.4 \pm \\
6.4\end{array}$ & $\dot{0} 001$ & $\begin{array}{l}24.1 \pm \\
12.5\end{array}$ & $\dot{0} 001$ & $\begin{array}{l}26.6 \pm \\
10.8\end{array}$ & $\begin{array}{l}<.001 \\
0.00\end{array}$ \\
\hline \multicolumn{9}{|c|}{ Chest CT with contrast enhancement $(n=12)$} \\
\hline \multirow[t]{2}{*}{$\begin{array}{l}\text { Attenuation } \\
\text { (HU) }\end{array}$} & lung & $\begin{array}{l}-718.9 \pm \\
139.4\end{array}$ & $\begin{array}{l}-718.7 \pm \\
144.0\end{array}$ & 0.989 & $\begin{array}{l}-720.5 \pm \\
145.4\end{array}$ & 0.989 & $\begin{array}{l}-719.1 \\
\pm 142.8\end{array}$ & 0.989 \\
\hline & $\begin{array}{l}\text { paraspinal } \\
\text { muscle }\end{array}$ & $\begin{array}{l}65.0 \pm \\
8.5\end{array}$ & $\begin{array}{l}64.6 \pm \\
5.8\end{array}$ & 0.425 & $\begin{array}{l}64.3 \pm \\
5.2\end{array}$ & 0.425 & $\begin{array}{l}62.6 \pm \\
6.8\end{array}$ & 0.425 \\
\hline Noise & & $\begin{array}{l}24.5 \pm \\
6.1\end{array}$ & $\begin{array}{l}12.6 \pm \\
3.8\end{array}$ & $\dot{0} 001$ & $\begin{array}{l}21.3 \pm \\
4.9\end{array}$ & 0.172 & $\begin{array}{l}14.8 \pm \\
4.7\end{array}$ & $\begin{array}{l}<.001 \\
0.00\end{array}$ \\
\hline CNR & lung & $\begin{array}{l}10.4 \pm \\
4.0\end{array}$ & $\begin{array}{l}17.8 \pm \\
8.1\end{array}$ & 0.010 & $\begin{array}{l}20.0 \pm \\
6.9\end{array}$ & 0.001 & $\begin{array}{l}21.4 \pm \\
8.6\end{array}$ & $\dot{0.001}$ \\
\hline SNR & lung & $9.6 \pm 3.7$ & $\begin{array}{l}16.4 \pm \\
7.6\end{array}$ & 0.011 & $\begin{array}{l}18.4 \pm \\
6.6\end{array}$ & 0.001 & $\begin{array}{l}19.8 \pm \\
8.1\end{array}$ & $\begin{array}{l}<.001 \\
0.00\end{array}$ \\
\hline \multicolumn{9}{|c|}{ Abdomen CT with contrast enhancement $(n=23)$} \\
\hline \multirow[t]{3}{*}{$\begin{array}{l}\text { Attenuation } \\
(\mathrm{HU})\end{array}$} & liver & $\begin{array}{l}131.4 \pm \\
28.4\end{array}$ & $\begin{array}{l}131.7 \pm \\
28.7\end{array}$ & 0.369 & $\begin{array}{l}125.9 \pm \\
39.1\end{array}$ & 0.369 & $\begin{array}{l}132.6 \pm \\
28.6\end{array}$ & 0.369 \\
\hline & aorta & $\begin{array}{l}185.7 \pm \\
45.2\end{array}$ & $\begin{array}{l}184.9 \pm \\
45.9\end{array}$ & 1.000 & $\begin{array}{l}185.3 \pm \\
45.1\end{array}$ & 0.227 & $\begin{array}{l}187.6 \pm \\
45.4\end{array}$ & 0.085 \\
\hline & $\begin{array}{l}\text { paraspinal } \\
\text { muscle }\end{array}$ & $\begin{array}{l}71.2 \pm \\
8.4\end{array}$ & $\begin{array}{l}71.3 \pm \\
6.6\end{array}$ & 1.000 & $\begin{array}{l}72.7 \pm \\
6.0\end{array}$ & 0.535 & $\begin{array}{l}71.1 \pm \\
6.2\end{array}$ & 1.000 \\
\hline
\end{tabular}

Note: Values are presented as the mean \pm standard deviation. ASIR-V 50, 50\% adaptive statistical iterative reconstruction-V; ASIR-V 100, 100\% ASIR-V; DLR-M, medium strength deep learning reconstruction; DLR-H, high strength DLR; CNR, contrast to noise ratio; SNR, signal to noise ratio. Image noise is based on standard deviation of paraspinal muscle attenuation. 


\begin{tabular}{|c|c|c|c|c|c|c|c|c|}
\hline \multirow{2}{*}{\multicolumn{2}{|c|}{$\begin{array}{l}\text { Parameters } \\
\text { Noise }\end{array}$}} & \multirow{2}{*}{$\begin{array}{l}\text { ASIR-V } \\
50 \\
19.9 \pm \\
3.7\end{array}$} & \multirow{2}{*}{$\begin{array}{l}\text { ASIR-V } 100 \\
\begin{array}{l}11.1 \pm \\
3.6\end{array}\end{array}$} & \multicolumn{2}{|c|}{ DLR-M } & \multicolumn{2}{|c|}{ DLR-H } & \multirow[b]{2}{*}{$\begin{array}{l}<.001 \\
0.00\end{array}$} \\
\hline & & & & $\hat{0}_{0.001}$ & $\begin{array}{l}16.3 \pm \\
3.1\end{array}$ & 0.002 & $\begin{array}{l}12.2 \pm \\
2.4\end{array}$ & \\
\hline \multirow[t]{2}{*}{ CNR } & liver & $3.2 \pm 1.7$ & $5.3 \pm 3.0$ & <. 001 & $3.2 \pm 2.4$ & 1.000 & $\begin{array}{l}4.9 \pm \\
2.5\end{array}$ & ¿. 001 \\
\hline & aorta & $5.3 \pm 2.2$ & $9.5 \pm 4.3$ & $\hat{0}_{0.001}$ & $5.8 \pm 1.9$ & 0.012 & $\begin{array}{l}8.0 \pm \\
2.9\end{array}$ & $\hat{0} .001$ \\
\hline \multirow[t]{2}{*}{ SNR } & liver & $6.8 \pm 2.1$ & $\begin{array}{l}11.5 \pm \\
3.9\end{array}$ & $<.001$ & $7.6 \pm 2.7$ & 0.583 & $\begin{array}{l}10.7 \pm \\
2.9\end{array}$ & $\hat{0}_{0.001}$ \\
\hline & aorta & $8.5 \pm 2.4$ & $\begin{array}{l}15.6 \pm \\
5.1\end{array}$ & $\begin{array}{l}<.001 \\
0.00\end{array}$ & $9.7 \pm 2.0$ & 0.002 & $\begin{array}{l}13.0 \pm \\
3.2\end{array}$ & $\begin{array}{l}<.001 \\
0.00\end{array}$ \\
\hline \multicolumn{9}{|c|}{$\begin{array}{l}\text { Note: Values are presented as the mean } \pm \text { standard deviation. ASIR-V } 50,50 \% \text { adaptive statistical } \\
\text { iterative reconstruction-V; ASIR-V } 100,100 \% \text { ASIR-V; DLR-M, medium strength deep learning } \\
\text { reconstruction; DLR-H, high strength DLR; CNR, contrast to noise ratio; SNR, signal to noise ratio. } \\
\text { Image noise is based on standard deviation of paraspinal muscle attenuation. }\end{array}$} \\
\hline
\end{tabular}

When compared with 50\% ASIR-V, high strength DLR was associated with noise reduction in non-contrast chest CT (33.0\%), contrast-enhanced chest CT (39.6\%), and contrast-enhanced abdomen CT (38.7\%) with increases in CNR at 149.1\%, 105.8\%, and 53.1\%, respectively, and increases in SNR at 148.6\%, 106.3\%, and $57.4 \%$, respectively (Fig. 2).

Medium strength DLR also showed decreased noise in abdomen $C T$, but no significant difference was found in noise in chest CT when compared with 50\% ASIR-V. Medium strength DLR showed better CNR and SNR in both non-contrast and contrast-enhanced chest CT; however, there was no significant difference in CNR and SNR in abdomen CT.

When compared with $100 \%$ ASIR-V, high strength DLR showed improved CNR in chest CT images without contrast enhancement by $24 \%$. However, there was no significant improvement in CNR in both chest CT and abdomen CT images with contrast enhancement (Supplementary Table 1).

We also analyzed the effects of body weight on noise reduction. In DLR group, the paraspinal muscle noise reduction was better in patients over $20 \mathrm{~kg}$ than in patients under $20 \mathrm{~kg}$ in both high strength group (noise: 16.9 in < $20 \mathrm{~kg}$ group vs. 13.3 in 20-60 kg group [p = 0.033] and $12.4 \mathrm{in}>60 \mathrm{~kg}$ group [p = 0.015$]$ ) and medium strength group (noise: 23.2 in $<20 \mathrm{~kg}$ group vs. 18.2 in $20-60 \mathrm{~kg}$ group [ $=0.028$ ] and 17.7 in $>60 \mathrm{~kg}$ group $[p=0.014])$. However, the noise was not different according to the body weight group in ASIR-V images.

\section{Qualitative image assessment}


The results of the subjective image quality analyses are summarized in Table 2 and Fig. 3. The subjective assessment of overall image quality and noise were also better on DLR images both on medium and high strength compared to $50 \%$ ASIR-V $(p<0.001)$. The agreement was moderate for overall image quality and good for noise in high strength DLR $(p<0.001)$. However, there was poor agreement in both image quality and noise in medium strength DLR $(p<0.001)$. There was no significant difference in motion or beam hardening artifacts between reconstruction methods with excellent interobserver agreement $(\mathrm{K}=0.944, p$ $<0.001$ ) (Fig. 4).

Table 2

Distribution of subjective image scoring for different reconstruction techniques by two pediatric radiologists.

\begin{tabular}{|c|c|c|c|c|c|}
\hline Parameter & & & Reviewer 1 & Reviewer 2 & *Agreement (K) \\
\hline \multirow[t]{4}{*}{ overall image quality } & $(1 / 2 / 3 / 4)$ & ASIR 50 & $0 / 47 / 4 / 0$ & $0 / 47 / 4 / 0$ & 0.728 \\
\hline & & ASIR 100 & $0 / 4 / 47 / 0$ & $0 / 0 / 51 / 0$ & 0 \\
\hline & & TF-M & $0 / 0 / 49 / 1$ & $0 / 0 / 49 / 1$ & -0.02 \\
\hline & & TF-H & $0 / 0 / 38 / 13$ & $0 / 0 / 35 / 16$ & 0.568 \\
\hline \multirow[t]{4}{*}{ noise } & $(1 / 2 / 3 / 4)$ & ASIR 50 & $0 / 47 / 4 / 0$ & $0 / 49 / 2 / 0$ & 0.297 \\
\hline & & ASIR 100 & $0 / 8 / 43 / 0$ & $0 / 0 / 51 / 0$ & 0 \\
\hline & & TF-M & $0 / 1 / 48 / 1$ & $0 / 0 / 51 / 0$ & 0 \\
\hline & & $\mathrm{TF}-\mathrm{H}$ & $0 / 1 / 34 / 16$ & $0 / 0 / 33 / 18$ & 0.62 \\
\hline \multirow[t]{4}{*}{ artifact } & $(1 / 2 / 3 / 4)$ & ASIR 50 & $0 / 11 / 40 / 0$ & $0 / 12 / 39 / 0$ & 0.944 \\
\hline & & ASIR 100 & $0 / 11 / 40 / 0$ & $0 / 12 / 39 / 0$ & 0.944 \\
\hline & & TF-M & $0 / 11 / 40 / 0$ & $0 / 12 / 39 / 0$ & 0.94 \\
\hline & & $\mathrm{TF}-\mathrm{H}$ & $0 / 11 / 40 / 0$ & $0 / 12 / 39 / 0$ & 0.944 \\
\hline
\end{tabular}

\section{Discussion}

Our study found that DLR can improve the quantitative and qualitative image quality in pediatric chest and abdomen CT relative to advanced IR technique, our standard 50\% ASIR-V. High strength DLR showed significant noise reduction with increased CNR and SNR. DLR also scored significantly better for image quality and noise subjectively. However, motion or beam hardening artifacts were not decreased with deep learning method, regardless of strength. 
The DLR algorithm has been developed for CT to remove image noise. The effect of DLR on image quality and its potential to lower patient radiation dose is being investigated. A phantom study demonstrated that DLR had superior noise, magnitude, noise texture, and spatial resolution [11]. Another study also showed that DLR improves the image quality through noise reduction and increased CNR without altering the image texture on abdomen CT [12]. They demonstrated that subjective diagnostic confidence was increased in all DLR images when compared with ASIR-V with a $30 \%$ blending factor, and the higher strength in DLR lowers the noise with increased sharpness [13]. The SNR and CNR values of high strength DLR images were higher than those of ASIR-V with 80 or $100 \%$ blending factor. Similar results were also reported in studies with different vendor systems and algorithms $[10,14,15]$.

DLR has been introduced to pediatric patients in a few studies of abdomen CT [16-18]. Lim et al. [16] studied a 5-year-old patient's phantom and pediatric abdomen CT exams using a vendor-neutral DLR technique and demonstrated similar image quality with hybrid IR technique. Brady et al. [17] used contrast-enhanced abdomen CT with DLR algorithm showing improved object detectability, reduced image noise, and high radiologist preference when compared to conventional IR images. About a $51 \%$ dose reduction using DLR was hypothesized on the basis of mathematical extrapolation from this retrospective study. Lee et al. [18] used DLR with low iodine concentration abdominal dual energy CT and showed decreased noise in DLR images without difference in CNR, overall image quality, and diagnostic quality of lesions. The CTDI ${ }_{\text {vol }}$ and total iodine administration was lower in dual energy CT with DLR. Both studies suggested that DLR has the potential to improve image quality and potentially reduce patient radiation dose. However, no study has evaluated the role of DLR in pediatric chest CT and the effect of DLR on image artifacts.

Our study shows similar results in noise reduction and quality improvement. High strength DLR was associated with noise reduction in non-contrast chest $\mathrm{CT}$, contrast-enhanced chest $\mathrm{CT}$, and contrastenhanced abdomen CT with increase in both CNR and SNR. The subjective assessment of overall image quality and noise were also better on DLR images both on medium and high strength DLR compared to $50 \%$ ASIR-V. Our study showed no significant difference in attenuation values of the organs in pediatric chest and abdomen. This result is comparable with a previous report with an adult population [12]. Therefore, we can use CT images with DLR for attenuation analyses such as emphysema index measurements.

Previous studies have focused on noise reduction and image quality improvement of DLR with little focus on artifacts. DLR scored better on artifacts than $30 \%$ ASIR-V images in a previous study [12]. Another study reported no DLR related image artifacts [14]. A prior study has reported more frequent distortion artifacts with DLR [22]. In our study, there was no significant difference in artifacts between reconstruction methods with excellent inter-observer agreement on artifacts. Mainly these artifacts were beam hardening artifacts from metal or dense contrast media in vessels. There were no significant distortion artifacts in our study. Depending on the purpose and input data of the DLR technology, the role of DLR may vary. Our DLR algorithm requires additional technologies for artifact reduction. 
Our study has limitations. First, the sample size of our retrospective study was small, and we could not evaluate lesion detectability or diagnostic accuracy. Second, the data is from a designated vendor's DLR algorithm. Third, the number of the patients with artifacts was not the majority of the patient population. Fourth, from the retrospective nature of our study, we could not compare images between FBP and DLR. Lastly, our study cannot suggest an estimated radiation dose reduction using DLR. Additional prospective studies with more patients are needed.

\section{Conclusions}

Compared with $50 \%$ ASIR-V, DLR improved the CT evaluation of pediatric chest and abdomen images with significant noise reduction. However, motion or beam hardening artifacts were not decreased by DLR, regardless of strength.

\section{List Of Abbreviations}

CT: computed tomography

DLR: deep learning reconstruction

IR: iterative reconstruction

ASIR-V :adaptive statistical iterative reconstruction $\mathrm{V}$

CNR: contrast to noise ratio

SNR: signal to noise

ANOVA: analysis of variance

FBP: filtered back projection

CTDI vol: CT dose index volume

SSDE: size-specific dose estimates

DLP: dose-length product

ED: effective dose

\section{Declarations}

\section{Ethics approval and consent to participate}

- Following the Declaration of Helsinki, the study was approved as a retrospective human study by the Institutional Review Board of Severance Hospital (No.4-2020-1363). The informed consent of patient, 
parents, or guardians was waived. All methods were carried out in accordance with relevant guidelines and regulations.

Consent for publication : not applicable

Availability of data and materials

- All data generated or analysed during this study are included in this published article and its supplementary information files

Competing interests: The authors have no competing interest to declare.

Funding: not applicable

Authors' contributions:

HY, MJL: study concept and design, data acquisition, data analysis and interpretation, and writing of manuscript. HY, JK, HJL, MJL: data analysis, interpretation and revision of manuscript for important intellectual content. All authors read and approved the final manuscript.

Acknowledgements: not applicable

\section{References}

1. Yanagawa M, Honda O, Kikuyama A, Gyobu T, Sumikawa H, Koyama M, Tomiyama N: Pulmonary nodules: effect of adaptive statistical iterative reconstruction (ASIR) technique on performance of a computer-aided detection (CAD) system-comparison of performance between different-dose CT scans. Eur J Radio/ 2012, 81(10):2877-2886.

2. Singh S, Kalra MK, Hsieh J, Licato PE, Do S, Pien HH, Blake MA: Abdominal CT: comparison of adaptive statistical iterative and filtered back projection reconstruction techniques. Radiology 2010, 257(2):373-383.

3. Bae S, Kim MJ, Yoon CS, Kim DW, Hong JH, Lee MJ: Effects of adaptive statistical iterative reconstruction on radiation dose reduction and diagnostic accuracy of pediatric abdominal CT. Pediatric radiology 2014, 44(12):1541-1547.

4. Yoon H, Kim MJ, Yoon CS, Choi J, Shin HJ, Kim HG, Lee MJ: Radiation dose and image quality in pediatric chest CT: effects of iterative reconstruction in normal weight and overweight children. Pediatric radiology 2015, 45(3):337-344.

5. Lee SH, Kim MJ, Yoon CS, Lee MJ: Radiation dose reduction with the adaptive statistical iterative reconstruction (ASIR) technique for chest CT in children: an intra-individual comparison. Eur J Radiol 2012, 81(9):e938-943.

6. Singh S, Kalra MK, Shenoy-Bhangle AS, Saini A, Gervais DA, Westra SJ, Thrall JH: Radiation dose reduction with hybrid iterative reconstruction for pediatric CT. Radiology 2012, 263(2):537-546. 
7. Goodenberger MH, Wagner-Bartak NA, Gupta S, Liu X, Yap RQ, Sun J, Tamm EP, Jensen CT:

Computed Tomography Image Quality Evaluation of a New Iterative Reconstruction Algorithm in the Abdomen (Adaptive Statistical Iterative Reconstruction-V) a Comparison With Model-Based Iterative Reconstruction, Adaptive Statistical Iterative Reconstruction, and Filtered Back Projection Reconstructions. J Comput Assist Tomogr 2018, 42(2):184-190.

8. Euler A, Solomon J, Marin D, Nelson RC, Samei E: A Third-Generation Adaptive Statistical Iterative Reconstruction Technique: Phantom Study of Image Noise, Spatial Resolution, Lesion Detectability, and Dose Reduction Potential. AJR Am J Roentgeno/ 2018, 210(6):1301-1308.

9. Padole A, Ali Khawaja RD, Kalra MK, Singh S: CT radiation dose and iterative reconstruction techniques. AJR Am J Roentgenol 2015, 204(4):W384-392.

10. Akagi M, Nakamura Y, Higaki T, Narita K, Honda Y, Zhou J, Yu Z, Akino N, Awai K: Deep learning reconstruction improves image quality of abdominal ultra-high-resolution CT. Eur Radio/2019, 29(11):6163-6171.

11. Greffier J, Hamard A, Pereira F, Barrau C, Pasquier H, Beregi JP, Frandon J: Image quality and dose reduction opportunity of deep learning image reconstruction algorithm for $\mathrm{CT}$ : a phantom study. Eur Radiol 2020, 30(7):3951-3959.

12. Jensen CT, Liu X, Tamm EP, Chandler AG, Sun J, Morani AC, Javadi S, Wagner-Bartak NA: Image Quality Assessment of Abdominal CT by Use of New Deep Learning Image Reconstruction: Initial Experience. AJR Am J Roentgenol 2020, 215(1):50-57.

13. Park C, Choo KS, Jung Y, Jeong HS, Hwang JY, Yun MS: CT iterative vs deep learning reconstruction: comparison of noise and sharpness. Eur Radiol 2020.

14. Shin YJ, Chang W, Ye JC, Kang E, Oh DY, Lee YJ, Park JH, Kim YH: Low-Dose Abdominal CT Using a Deep Learning-Based Denoising Algorithm: A Comparison with CT Reconstructed with Filtered Back Projection or Iterative Reconstruction Algorithm. Korean J Radio/ 2020, 21(3):356-364.

15. Singh R, Digumarthy SR, Muse VV, Kambadakone AR, Blake MA, Tabari A, Hoi Y, Akino N, Angel E, Madan $\mathrm{R}$ et al: Image Quality and Lesion Detection on Deep Learning Reconstruction and Iterative Reconstruction of Submillisievert Chest and Abdominal CT. AJR Am J Roentgeno/ 2020, 214(3):566573.

16. Lim WH, Choi YH, Park JE, Cho YJ, Lee S, Cheon JE, Kim WS, Kim IO, Kim JH: Application of VendorNeutral Iterative Reconstruction Technique to Pediatric Abdominal Computed Tomography. Korean J Radiol 2019, 20(9):1358-1367.

17. Brady SL, Trout AT, Somasundaram E, Anton CG, Li Y, Dillman JR: Improving Image Quality and Reducing Radiation Dose for Pediatric CT by Using Deep Learning Reconstruction. Radiology 2020:202317.

18. Lee S, Choi YH, Cho YJ, Lee SB, Cheon JE, Kim WS, Ahn CK, Kim JH: Noise reduction approach in pediatric abdominal CT combining deep learning and dual-energy technique. Eur Radiol 2020.

19. Boone J SK, Cody D et al: Size-specific dose estimates (SSDE) in pediatric and adult body CT examinations. 2011. 
20. Deak PD, Smal Y, Kalender WA: Multisection CT protocols: sex- and age-specific conversion factors used to determine effective dose from dose-length product. Radiology 2010, 257(1):158-166.

21. Greffier J, Pereira F, Macri F, Beregi J-P, Larbi A: CT dose reduction using Automatic Exposure Control and iterative reconstruction: A chest paediatric phantoms study. Physica Medica 2016, 32(4):582589.

22. Nam JG, Hong JH, Kim DS, Oh J, Goo JM: Deep learning reconstruction for contrastenhanced CT of the upper abdomen: similar image quality with lower radiation dose in direct comparison with iterative reconstruction. Eur Radio/ 2021.

\section{Figures}
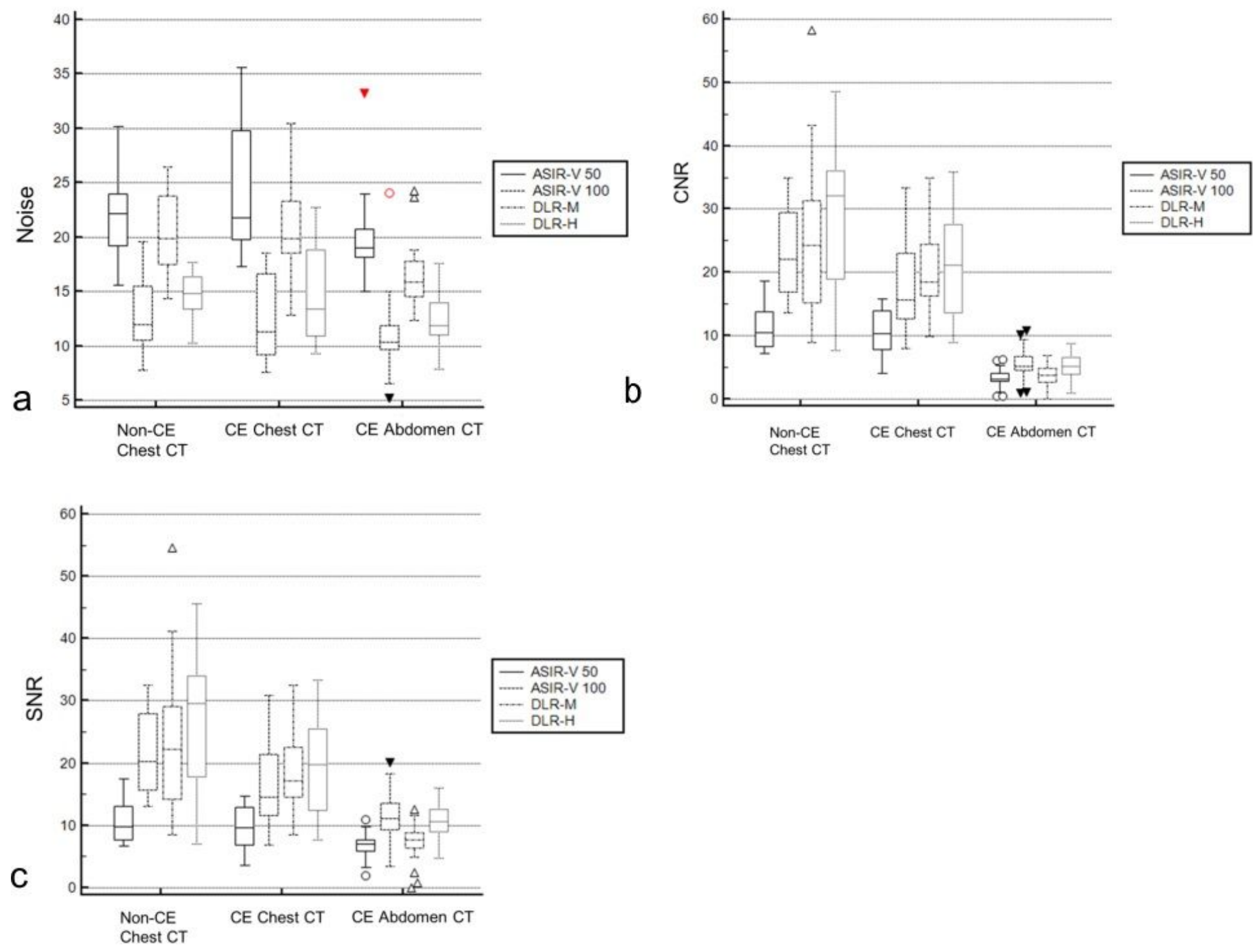

Figure 1

Box-and-whisker plots of quantitative pediatric CT image analyses with different reconstruction techniques. When compared with $50 \%$ adaptive statistical iterative reconstruction-V (ASIR-V), high 
strength deep learning reconstruction (DLR-H) was associated with (A) noise reduction, (B) better contrast to noise ratio (CNR), and (C) better signal to noise ratio (SNR). ASIR-V 50,50\% adaptive statistical iterative reconstruction-V; ASIR-V 100, 100\% ASIR-V; DLR-M, medium strength deep learning reconstruction; DLR-H, high strength DLR; CE, contrast-enhanced.

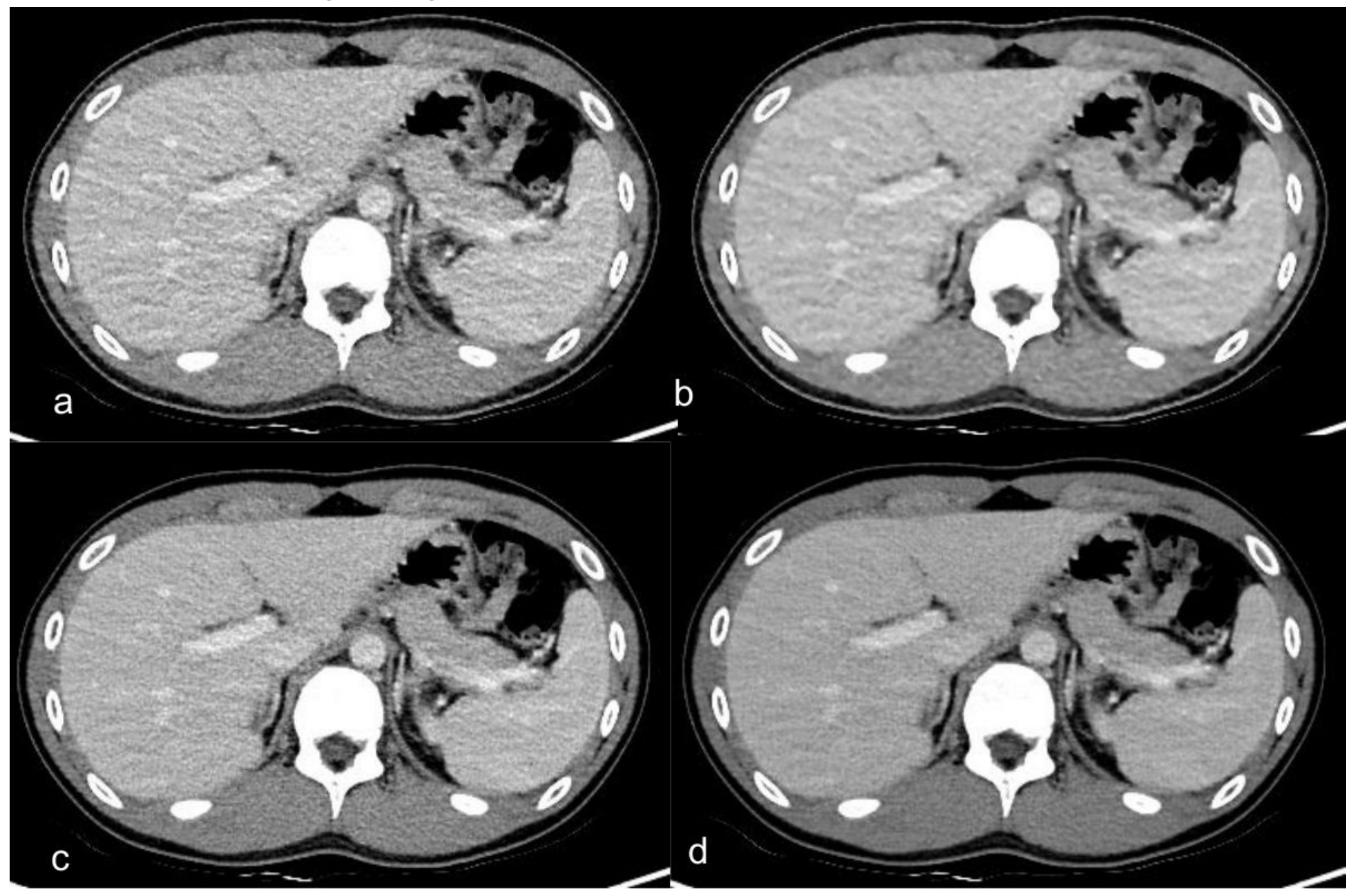

Figure 2

Abdomen CT images with contrast enhancement in a 15-year-old boy who had abdominal pain with a BMl of $19.9 \mathrm{~kg} / \mathrm{m} 2$. (A-D) Axial contrast-enhanced CT images of the same anatomical location show image quality comparison between (A) standard $50 \%$ adaptive statistical iterative reconstruction-V (50\% ASIR-V), (B) 100\% ASIR-V, (C) medium-strength deep learning image reconstruction (DLR-M), and (D) highstrength deep learning image reconstruction (DLR-H). Contrast to noise ratio (CNR) in the liver was 2.18 in $50 \%$ ASIR-V, 2.84 in 100\% ASIR-V, 3.03 in DLR-M, 3.88 in DLR-H. 


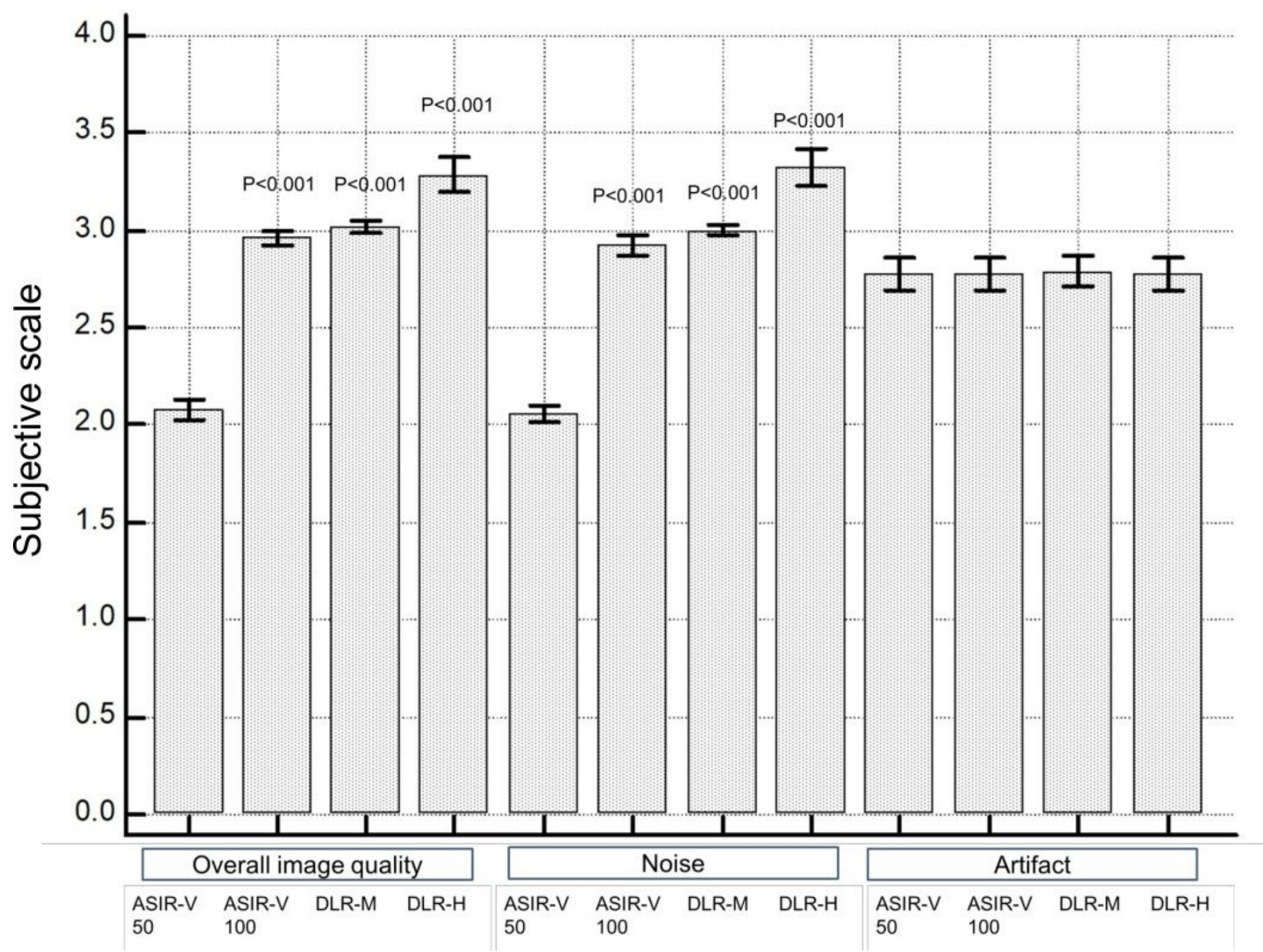

Figure 3

Qualitative image analysis of chest and abdomen CT from different reconstruction techniques. The fourpoint scale was used as follows; superior (4), average (3), suboptimal (2), unacceptable (1). Deep learning reconstruction (DLR) showed better overall image quality and noise compared with $50 \%$ adaptive statistical iterative reconstruction-V (ASIR-V); however, artifacts were not different between different reconstruction techniques. ASIR-V 50,50\% adaptive statistical iterative reconstruction-V; ASIR-V 100, 100\% ASIR-V; DLR-M, medium strength deep learning reconstruction; DLR-H, high strength DLR. 


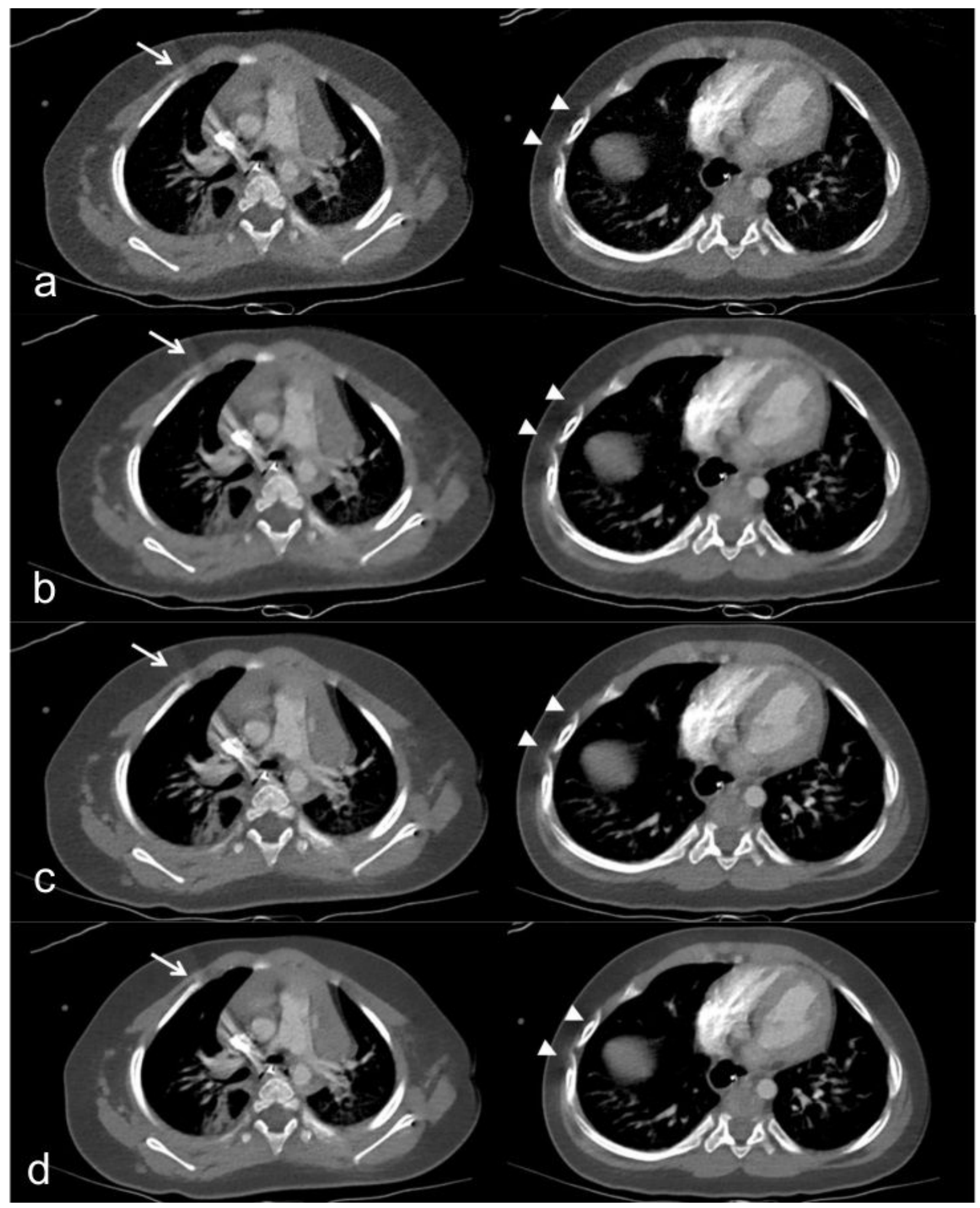

Figure 4

Chest CT images with mediastinal window of a 1-year-old girl who had a cough. (A-D) Axial contrastenhanced CT images with (A) standard 50\% adaptive statistical iterative reconstruction-V (50\% ASIR-V), (B) $100 \%$ ASIR-V, (C) medium-strength deep learning image reconstruction (DLR-M), and (D) high-strength deep learning image reconstruction (DLR-H) show no difference in beam hardening artifacts due to dense contrast material in the superior vena cava (arrow) and motion artifacts in the bilateral ribs (arrow heads), 
resulting in lower reader scores for artifacts. Both readers thought the image was suboptimal. Contrast to noise ratio (CNR) of the lung was 4.8 in 50\% ASIR-V, 12.0 in 100\% ASIR-V, 13.0 in DLR-M, and 15.0 in DLR$\mathrm{H}$.

\section{Supplementary Files}

This is a list of supplementary files associated with this preprint. Click to download.

- Supplementarytable1.docx 